\title{
TransparentPEG-Fibrin Gel as a Flexible Tool for Cell Encapsulation
}

DOI: $10.17691 / \mathrm{stm} 2018.10 .1 .08$

Received October 3, 2017

A.I. Shpichka, PhD, Senior Research Fellow';

V.A. Revkova, Research Fellow2;

N.A. Aksenova, PhD, Senior Research Fellow ${ }^{1,3}$;

G.M. Yusubalieva, MD, PhD, Senior Research Fellow2;

V.A. Kalsin, Research Fellow;

E.F. Semenova, PhD, Professor';

Y. Zhang, MD, PhD, Assistant Professor ${ }^{5}$;

V.P. Baklaushev, MD, DSc, Chief Scientific Officer²;

P.S. Timashev, DSc, Director of the Institute for Regenerative Medicine ${ }^{1}$; Leading Researcher ${ }^{6}$; Researcher ${ }^{3}$

II.M. Sechenov First Moscow State Medical University, 8/2 Trubetskaya St., Moscow, 119991, Russia;

${ }^{2}$ Federal Research Clinical Center, Federal Medico-Biological Agency of Russia, 28 Orekhovy Blvd.,

115682, Moscow, Russia;

${ }^{3}$ Semenov Institute of Chemical Physics, Russian Academy of Sciences, 4 Kosygina St., Moscow, 119991, Russia;

${ }^{4}$ Penza State University, 40 Krasnaya St., Penza, 440018, Russia;

${ }^{5}$ Institute for Regenerative Medicine, Wake Forest University School of Medicine, Medical Center Blvd.,

Winston-Salem, NC 27157, USA:

${ }^{6}$ Institute of Photonic Technologies, Research Center "Crystallography and Photonics",

Russian Academy of Sciences, 2 Pionerskaya St., Moscow, Troitsk, 142190, Russia

The aim of this study was to modify the chemical structure and to optimize the composition of the fibrin gel for effective cell encapsulation.

Materials and Methods. We prepared PEGylated fibrin gels using different fibrinogen concentrations (25-50 mg/ml) and PEGfibrinogen molar ratio 10:1 and 5:1 and characterized them via Fourier transform infrared spectroscopy and differential scanning calorimetry. Within the gels, we encapsulated primary culture of fibroblasts and analyzed using light and laser confocal microscopy.

Results. PEGylation of fibrinogen allowed us to achieve the gel transparency and preserve its biocompatibility. We revealed that the gel prepared from PEGylated 5:1 fibrinogen $(25 \mathrm{mg} / \mathrm{ml})$ provided the most favorable microenvironment for spreading, growth, and proliferation of fibroblasts. This PEG-fibrin gel can be used for encapsulation of different cell types that is essential for various approaches in tissue engineering and diagnostic systems.

Key words: fibrin gel; cell encapsulation; PEGylation; matrix; fibroblasts.

How to cite: Shpichka A.I., Revkova V.A., Aksenova N.A., Yusubalieva G.M., Kalsin V.A., Semenova E.F., Zhang Y., Baklaushev V.P., Timashev P.S. Transparent PEG-fibrin gel as a flexible tool for cell encapsulation. Sovremennye tehnologii v medicine 2018; 10(1): 64, https:/l doi.org/10.17691/stm2018.10.1.08

\section{Introduction}

One of the key issues in tissue engineering is the support of 3D growth of cells. For several decades, extracellular matrices derived from different sources have been used to achieve this goal. Such materials include collagen, fibronectin, laminin, and reconstituted basement membranes (e.g. Matrigel) [1]. Among them, fibrin is one of the most common biomaterials used for cell encapsulation due to its good biocompatibility

Corresponding author: Anastasia I. Shpichka, e-mail: ana-shpichka@yandex.ru 
and easy handling. It is formed from fibrinogen, which circulates in blood vessels, by catalytic cleavage caused by thrombin [2]. There are numerous approaches to modify fibrin physical properties. For example, we can regulate its density by changing $\mathrm{pH}$, fibrinogen, and calcium ion concentrations, and thrombin and fibrinogen ratio and adding factor XIII [3]. However, fibrin gel has two main limitations: high degradation rate and opacity [2]. To overcome these obstacles, we can use chemical modification, e.g. PEGylation. This method permits us to conjugate protein with functionalized polyethylene glycol (PEG). PEGylation of fibrinogen allows the control of gel physical properties and degradation rate [4]. Therefore, it enables the long-term cell cultivation [5].

This study aims to was to modify the chemical structure and to optimize the composition of the fibrin gel for effective cell encapsulation.

\section{Materials and Methods}

Preparation of fibrinogen and thrombin solutions. We dissolved lyophilized fibrinogen and thrombin from bovine plasma (Sigma-Aldrich, Germany) in sterile phosphate buffered saline (PBS) at a concentration of 50 and $100 \mathrm{U} / \mathrm{ml}$, respectively. Before their use, protein and enzyme solutions were stored at $-20^{\circ} \mathrm{C}$. Fibrinogen concentrations of 25,30 , and $40 \mathrm{mg} / \mathrm{ml}$ were prepared before cell encapsulation.

Fibrinogen modification. Fibrinogen was covalently bonded to PEG using O,O'-bis[2-(N-succinimidylsuccinylamino)ethyl]polyethylene glycol (PEG-NHS; Sigma-Aldrich, Germany). We diluted PEG-NHS in PBS at a concentration of $1.5 \mathrm{mg} / \mathrm{ml}$ and added it to fibrinogen solutions in molar ratios of 10:1 and 5:1 (PEGNHS:fibrinogen). The reaction mixture was placed into a thermostat $\left(37^{\circ} \mathrm{C}\right)$ for $2 \mathrm{~h}$.

Fourier transform infrared spectroscopy and differential scanning calorimetry analysis. We lyophilized the solutions before testing. Fourier transform infrared (FTIR) spectra were obtained using a Spotlight 400N FTNIR Imaging System (Perkin Elmer, USA). Differential scanning calorimetry (DSC) was performed via a STA 6000 simultaneous thermal analyzer (Perkin Elmer, USA). The sample weight was $10 \mathrm{mg}$. Polymer destruction was carried out in a nitrogen medium at a gas flow rate of $40 \mathrm{ml} / \mathrm{min}$ and linear heating rate of $20^{\circ} \mathrm{C} / \mathrm{min}$. Mass losses were registered to $3-10 \mathrm{mg}$; the relative errors of temperature and thermal effect measuring were $\pm 1.5^{\circ} \mathrm{C}$ and $\pm 2 \%$, respectively. Destruction process was described via temperature dependence of mass loss (thermal gravimetric analysis) and thermal effects (differential thermal analysis, DTA). We revealed the initial mass loss temperature for both modified and nonmodified fibrinogen.

Primary culture of fibroblasts. The fibroblast primary cultures were obtained by a biopsy of the behind-theear skin regions. The biopsy was performed from a healthy volunteer who signed the informed consent. The
$2 \times 1 \times 1 \mathrm{~mm}$ biopsy specimen was washed in Dulbecco's phosphate-buffered saline supplemented with $1 \mathrm{X}$ antibiotic-antimycotic solution (Gibco, USA). Then, the dermis was dissected from the rest of the skin (epidermis, subcutaneous tissue, vascular structures) using scalpel and forceps. The resulting dermis preparation was washed with DMEM/F12, mechanically minced, then dissociated in a solution of collagenase I (Gibco, USA) $(1 \mathrm{mg} / \mathrm{ml}$ ) in the DMEM/F12 culture medium with a glucose concentration of $1 \mathrm{~g} / \mathrm{L}$. The preparation was incubated for $3 \mathrm{~h}$ using an orbital shaker with constant rocking and a temperature of $37^{\circ} \mathrm{C}$. It was then washed from the enzyme with the culture medium, passed through a filter (100 $\mu \mathrm{m}$; SPL Life Science, Korea) to remove the fibers and precipitated by centrifugation. The cell pellet was suspended in the complete DMEM/F12 (Gibco, USA) growth medium containing $1 \mathrm{~g} / \mathrm{L}$ glucose, $10 \%$ fetal bovine serum, $1 \mathrm{X}$ GlutaMAX solution, and $1 \mathrm{X}$ antibiotic-antimycotic solution, and seeded in a culture flask (Eppendorf), based on the coverage of $1 \cdot 10^{6}$ cells/ $\mathrm{cm}^{2}$ and cultured in a $\mathrm{CO}_{2}$ incubator at $5 \% \mathrm{CO}_{2}, 5 \% \mathrm{O}_{2}$ at $37^{\circ} \mathrm{C}$ until the $100 \%$ confluence of the monolayer was reached, then the cells were dissociated with $0.25 \%$ trypsin-EDTA (Gibco, USA) and the resulting suspension was used to prepare the hydrogel cell preparations.

Cell encapsulation. Cell suspension $(50 \mu \mathrm{l})$ containing $3 \cdot 10^{4}$ cells was distributed in $150 \mu$ l of fibrinogen solution. The sufficient volume of thrombin stock solution was added (fibrinogen to thrombin ratio: $1 \mathrm{mg}$ to $0.2 \mathrm{U}$ ). This mixture immediately formed a gel and was cultured in a $\mathrm{CO}_{2}$ incubator for 3-7 days.

Light microscopy was performed using a Primovert inverted microscope (Carl Zeiss, Germany).

Immunocytochemical analysis. Expression of markers in fibroblasts and in the fibrin gel was assessed by immunocytochemical analysis. The staining was performed in $35 \mathrm{~mm}$ dishes with a central coverslip hole (SPL Life Science, Korea). The growth medium was removed before the fixation and the gels were washed with PBS. The cell-containing hydrogels were fixed by addition of $4 \%$ buffered paraformaldehyde solution with $0.1 \%$ saponin for 30 min followed by washing with PBS with $0.1 \%$ Triton $\mathrm{X} 100,0.1 \%$ Tween 20 , and $0.5 \%$ normal goat medium (working buffer). As primary antibodies, we used antibodies to BCL2, collagen types I-IV, CD51/61, SMI-1, Ki67, vimentin. As secondary antibodies, we used goat anti-mouse and goat-anti-rabbit antibodies labeled with Alexa Fluor 488 and Alexa Fluor 633 (Alexa Fluor 488 goat anti-mouse IgG $(\mathrm{H}+\mathrm{L})$ and Alexa Fluor 633 goat anti-rabbit IgG $(\mathrm{H}+\mathrm{L})$; all 1:400; Life Technologies, USA). The cell nuclei were stained with Hoechst. To measure the proliferative activity of the cells in the fibrin gel, we applied two approaches: (1) using sections stained with antibodies to Ki67, we determined the proliferation index; (2) the use of a kit for staining proliferating cells ClickiT Plus EdU Alexa 647 Imaging Kits (Life Technologies, USA). Briefly: 2 days prior to fixation, EdU in the concentration of $5 \mu \mathrm{M}$ was added to the hydrogels with 
the cells, then the preparations were fixed, and the cells which passed the S-phase of mitosis and incorporated EdU into the nuclear DNA were detected via the manufacturer protocol. The results of the fluorescence studies were analyzed using a Nikon A1 laser scanning confocal microscope (Nikon Co., Japan). The proliferation index was calculated as the percentage of Ki67/EdUpositive nuclei in respect to the total number of Hoechststained nuclei. All the calculations were performed in 5 repeats with 10 fields of view in each repeat.

\section{Results and Discussion}

Hydrogels prepared from PEGylated 5:1 and 10:1 fibrinogen were transparent compared to turbid pure fibrin gel. The fibrinogen chemical modification was proven via FT-IR analysis. Figure 1 demonstrates the corresponding FT-IR spectra of non-modified and

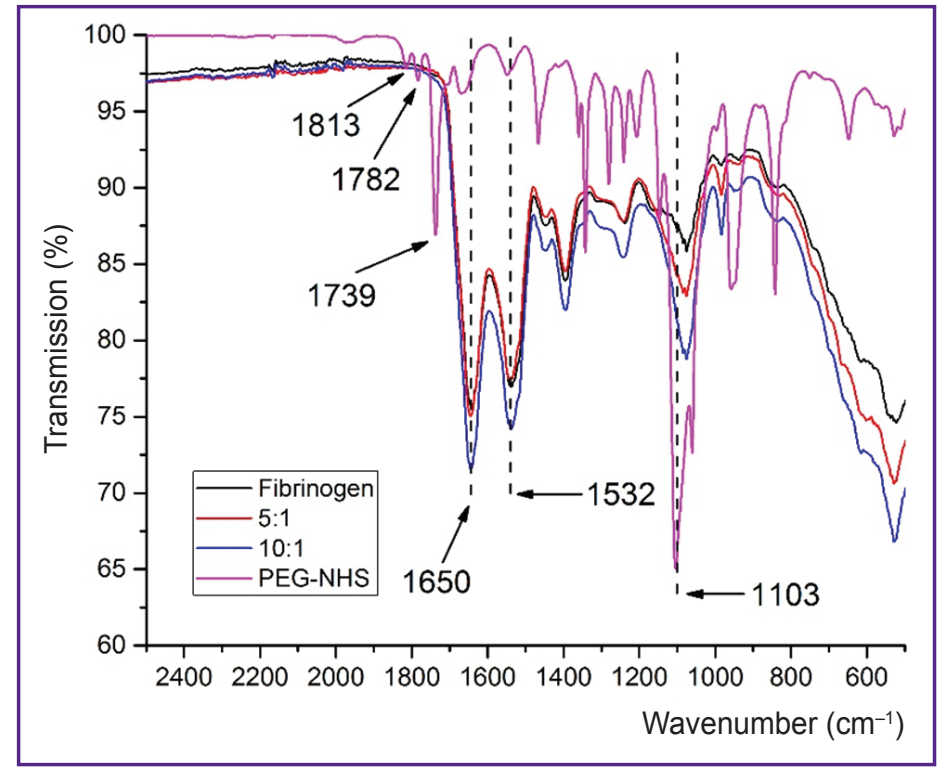

Figure 1. Fourier transform infrared spectra of pure fibrinogen, NHS-functionalized polyethylene glycol (PEG-NHS) and PEGylated fibrinogen with molar ratios 5:1 and 10:1 modified $(5: 1,10: 1)$ fibrinogen and NHS-functionalized PEG. PEG-NHS has a specific succinimidyl ester triplet band $\left(1739,1782\right.$, and $\left.1813 \mathrm{~cm}^{-1}\right)$, which was not revealed in the modified fibrinogen samples due to the reaction with primary amino groups caused by imide ringopening [6, 7]. These samples had an increasing band at $1103 \mathrm{~cm}^{-1}$, which indicates the insertion of PEG-derived (C-O) units and varies depending on molar ratio. Similar peaks were revealed for amide I and amide II bands at 1650 and $1532 \mathrm{~cm}^{-1}$, which are present in PEG-NHS. The increasing succinimidyl ester and amide peaks characterized the successful fibrinogen modification.

Figure 2 shows the temperature dependences of mass loss and thermal effects assessed for non-modified and modified fibrinogen. We revealed no significant changes in thermal stability for PEGylated 5:1 fibrinogen compared to non-modified one. However, PEGylated 10:1 fibrinogen had the decrease in the initial mass loss temperature from 270 to $250^{\circ} \mathrm{C}$. This might be caused by the low thermal stability of macroradicals, which were formed due to thermal exposure and contained numerous PEG fragments. Moreover, fibrinogen modified 10:1 was more hydrated than others that was revealed because of a drop in a sample weight in a range of temperature from 50 to $90^{\circ} \mathrm{C}$. Figure 2 also demonstrates that polycondensed structures (carbon residue) were formed due to macroradical interaction at $400-600^{\circ} \mathrm{C}$ and their content was app. $50 \%$ of sample weight. Such high carbon residue content is common for cross-linked systems [8]. However, as we can observe at DTA curves, the destruction process of all fibrinogen types occurred without remarkable stages.

During cell cultivation, gels retained their shape and external properties. Within them, fibroblasts were uniformly distributed (Figure 3). When they were encapsulated using both fibrinogen (PEGylated 5:1 and 10:1) concentrations 25 and $30 \mathrm{mg} / \mathrm{ml}$, the cells had a spindle shape and formed a network. However, within PEGylated 5:1 fibrin gel with $25 \mathrm{mg} / \mathrm{ml}$, the cells proliferated more actively than those within other gels, and no conglomerates

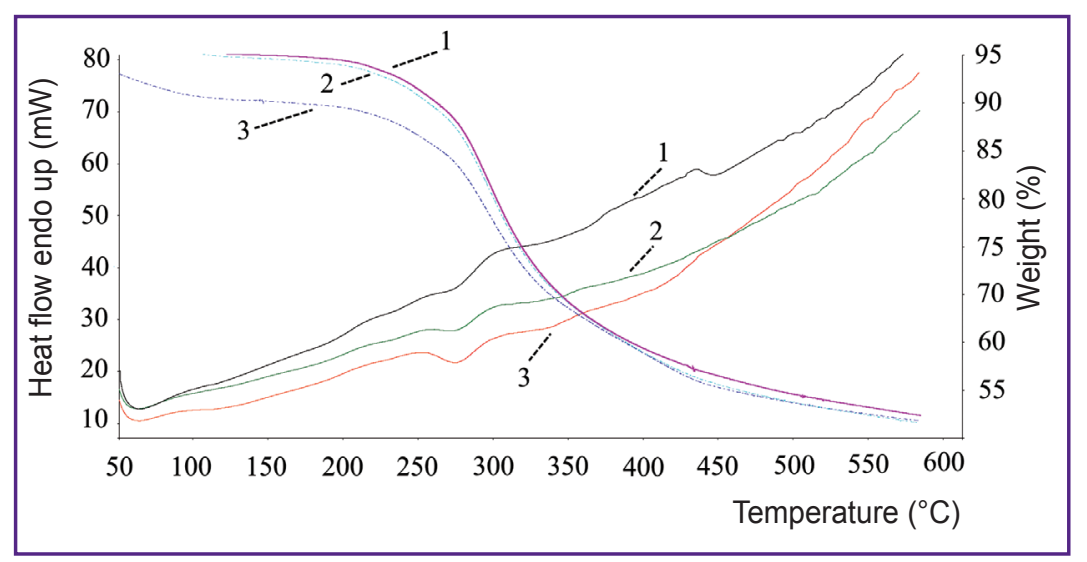

Figure 2. Temperature dependence of mass loss and thermal effects: 1 - pure, 2 - 5:1 PEGylated, and 3 - 10:1 PEGylated fibrinogen 


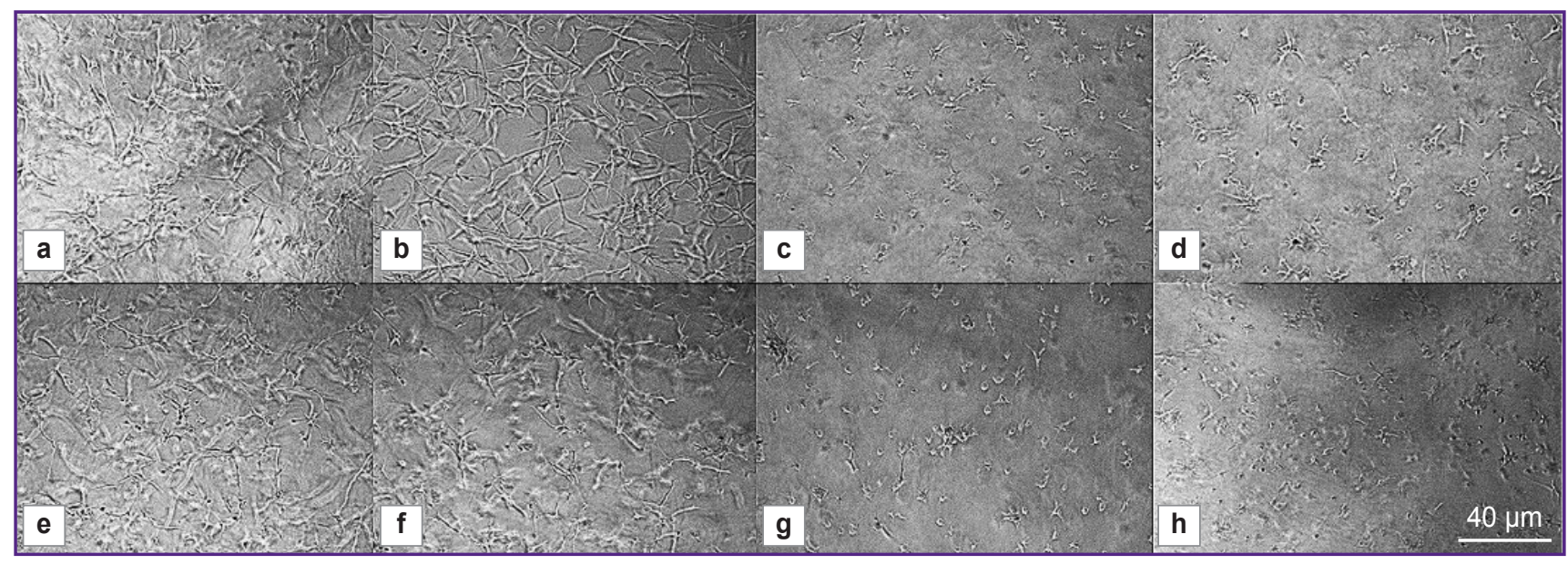

Figure 3. Microscopic study of fibroblast culture encapsulated within the gels prepared with different concentrations of modified fibrinogen (day 4):

(a)-(d) PEGylated 5:1 fibrin gel (fibrinogen concentration, $\mathrm{mg} / \mathrm{ml}$ : (a) 25, (b) 30, (c) 40, (d) 50); (e)-(h) PEGylated 10:1 fibrin gel (fibrinogen concentration, mg/ml: (e) 25, (f) 30, (g) 40, (h) 50)
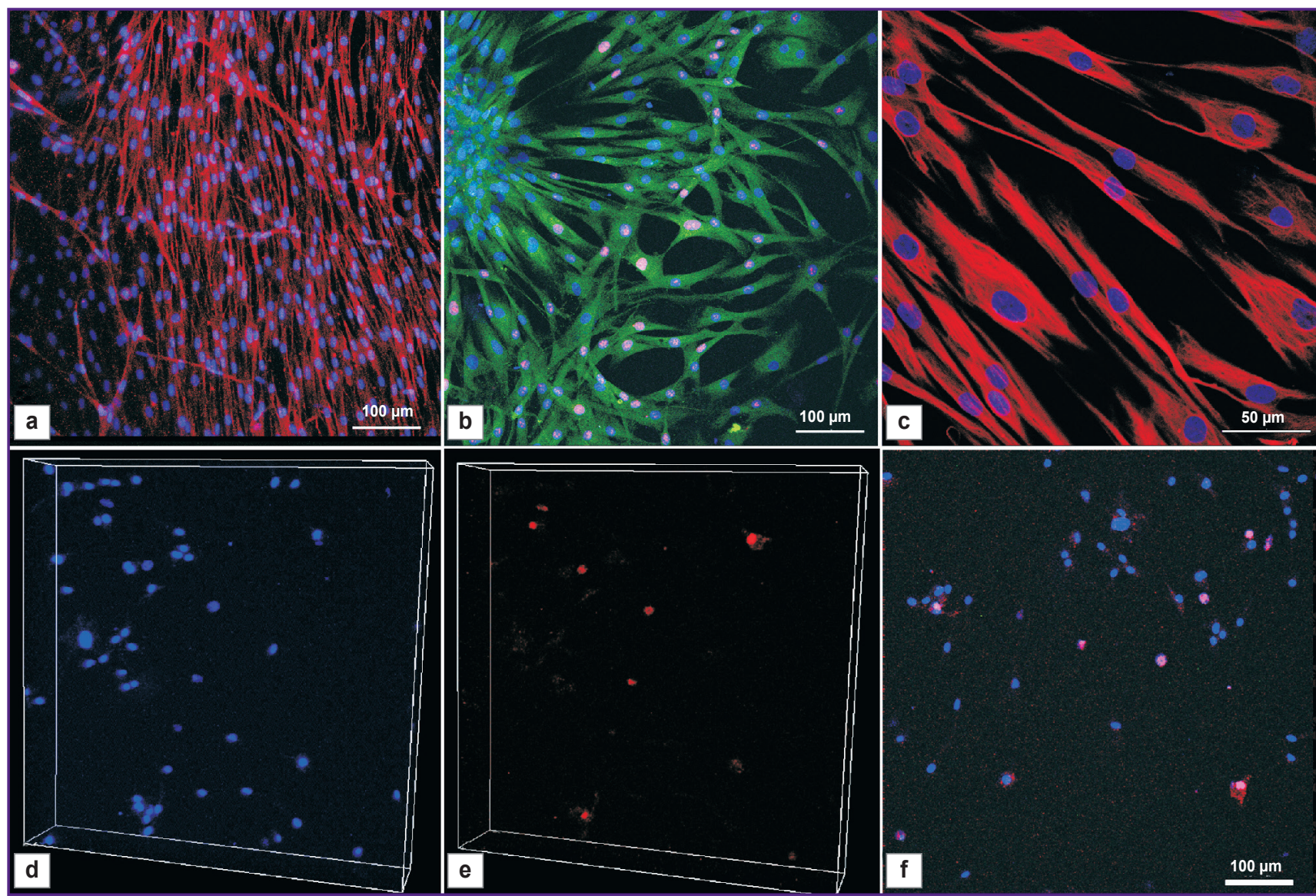

Figure 4. Immunocytochemical study of primary fibroblast culture in fibrin hydrogel after 2 weeks of cultivation:

(a) BCL2 (rabbit polyclonal antibodies), secondary antibodies - Alexa Fluor 633 goat anti-rabbit. (b) Green channel: fibronectin (mouse monoclonal antibodies), secondary antibodies - Alexa Fluor 488 goat anti-mouse; Red channel: Ki67 (rabbit polyclonal antibodies), secondary antibodies - Alexa Fluor 633 goat anti-rabbit. (c) Green channel: vimentin (rabbit polyclonal antibodies), secondary antibodies - Alexa Fluor 488 goat anti-rabbit. (d)-(f) Proliferation assay by Click-iT Plus EdU Alexa Fluor 647 Imaging Kits: (d) 3D, Hoechst; (e) 3D, Alexa Fluor 647; (f) merged maximal projection 
of the cells or products of their apoptosis were observed (Figure 3 (a)). At higher PEGylation molar ratio and fibrinogen concentrations, the cells were spherical and hardly formed outgrowths (Figure 3 (c), (d), (g), (h)). This data corresponded with previously reported results with human umbilical vein endothelial cells and adiposederived stem cells [9]. Thus, for the following analysis, we chose a gel with $25 \mathrm{mg}$ PEGylated 5:1 fibrinogen per $1 \mathrm{ml}$.

The immunocytochemical analysis showed that fibroblasts in the hydrogel expressed fibronectin, BCL2, vimentin (Figure 4 (a)-(c)) and did not express integrin $\alpha \mathrm{V} \beta 3$, responsible for adhesion to fibrin. The expression of collagen types I-IV was also not found, probably, due to insufficient cultivation time.

Immunostaining with Ki67 antibodies and Click-iT Plus EdU Alexa 647 Imaging Kits (Life Technologies, USA) showed that fibroblasts in the gel actively proliferated. The proliferation index in the hydrogel preparations measured via Ki67 oscillated from 3 to $5 \%$ averaging at $4.5 \pm 1.5 \%$. When stained with Click-iT Plus EdU Alexa 647, the proliferation index was somewhat higher (since in this case all the cells passed the S-phase of mitosis after EdU addition are taken into account) and was $8.2 \pm 2.5 \%$ (Figure 4 (d)-(f)). In total, the obtained data testified that the optimal conditions for the growth and proliferation of primary dermal fibroblasts are created in the developed fibrin gels. Fibroblasts expressed specific markers and actively proliferated.

The mechanical cell-environment interactions provide various signals that control cell shape and function [10]. The presented fibrin gel diversity caused different cell adhesion in 3D [4, 11-13]. We used PEGylation to control the gel stability for long-term cell cultivation. Using the optimal parameters for covalent PEG binding to a protein molecule, we do not change the conjugate activity (e.g., receptor affinity, enzyme) [14]. PEGylation of fibrinogen had no influence on its ability to undergo enzymatic thrombin cleavage and cross-linking. We tested different PEG-fibrinogen molar ratios and fibrinogen concentrations to reveal the optimal gel composition. We selected 10:1 and 5:1 ratios to modify fibrinogen and its concentrations of $25,30,40$, and $50 \mathrm{mg} / \mathrm{ml}[5,15]$. PEGylated fibrin gels remained transparent after adding thrombin. This is a great physical advantage, which allows us to analyze cells cultured within the hydrogel via a microscope. The behavior of the cells encapsulated correlated with the hydrogel density caused by the fibrinogen concentration and PEG-fibrin molar ratio.

\section{Conclusion}

PEGylation of fibrinogen allowed us to achieve the gel transparency and preserve its biocompatibility. We revealed that the gel prepared from PEGylated $5: 1$ fibrinogen $(25 \mathrm{mg} / \mathrm{ml})$ provided the most favorable microenvironment for spreading, growth, and proliferation of fibroblasts. This PEG-fibrin gel can be used for encapsulation of different cell types that is essential for various approaches in tissue engineering and diagnostic systems.

Acknowledgements. This work was supported by the Russian Foundation for Basic Research (17-02-01248; hydrogel preparation and fibrinogen modification), the Federal Agency of Scientific Organizations (Agreement No.007-GZ/Ch3363/26; FT IR and DSC analysis), and the Russian Science Foundation (16-15-10432; fibroblast isolation, cell encapsulation, light microscopy, immunocytochemical staining and analysis).

Conflict of interest. The authors declare the absence of a conflict of interest.

\section{References}

1. Prewitz M.C., Seib F.P., von Bonin M., Friedrichs J., Stißel A., Niehage C., Müller K., Anastassiadis K., Waskow C., Hoflack B., Bornhäuser M., Werner C. Tightly anchored tissuemimetic matrices as instructive stem cell microenvironments. Nat Methods 2013; 10(8): 788-794, https://doi.org/10.1038/ nmeth.2523.

2. Shaikh F.M., Callanan A., Kavanagh E.G., Burke P.E., Grace P.A., McGloughlin T.M. Fibrin: a natural biodegradable scaffold in vascular tissue engineering. Cells Tissues Organs 2008; 188(4): 333-346, https://doi.org/10.1159/000139772.

3. Rowe S.L., Lee S., Stegemann J.P. Influence of thrombin concentration on the mechanical and morphological properties of cell-seeded fibrin hydrogels. Acta Biomater 2007; 3(1): 59-67, https://doi.org/10.1016/j. actbio.2006.08.006.

4. Bryant S.J., Durand K.L., Anseth K.S. Manipulations in hydrogel chemistry control photoencapsulated chondrocyte behavior and their extracellular matrix production. $J$ Biomed Mater Res 2003; 67A(4): 1430-1436, https://doi.org/10.1002/ jbm.a.20003.

5. Galler K.M., Cavender A.C., Koeklue U., Suggs L.J., Schmalz G., D'Souza R.N. Bioengineering of dental stem cells in a PEGilated fibrin gel. Regen Med 2011; 6(2): 191-200, https://doi.org/10.2217/rme.11.3.

6. Touahir L., Chazalviel J.-N., Sam S., Moraillon A., Henry de Villeneuve C., Allongue P., Ozanam F., GougetLaemmel A.C. Kinetics of activation of carboxyls to succinimidyl ester groups in monolayers grafted on silicon: an in situ real-time infrared spectroscopy study. J Phys Chem C 2011; 115(14): 6782-6787, https://doi.org/10.1021/ jp200150m.

7. Frey B.L., Corn R.M. Covalent attachment and derivatization of poly(l-lysine) monolayers on gold surfaces as characterized by polarization-modulation FT-IR spectroscopy. Anal Chem 1996; 68(18): 3187-3193, https://doi.org/10.1021/ ac9605861

8. Hu X., Kaplan D., Cebe P. Determining beta-sheet crystallinity in fibrous proteins by thermal analysis and infrared spectroscopy. Macromolecules 2006; 39(18): 6161-6170, https://doi.org/10.1021/ma0610109.

9. Shpichka A.I., Koroleva A.V., Deiwick A., Timashev P.S. Semenova E.F., Moiseeva I.Y., Konoplyannikov M.A., Chichkov B.N. Evaluation of the vasculogenic potential of hydrogels based on modified fibrin. Cell and Tissue 
Biology 2017; 11(1): 81-87, https://doi.org/10.1134/ s1990519x17010126.

10. Hoffman B.D., Grashoff C., Schwartz M.A. Dynamic molecular processes mediate cellular mechanotransduction. Nature 2011; 475(7356): 316-323, https://doi.org/10.1038/ nature10316.

11. Bensaïd W., Triffittb J.T., Blanchata C., Oudina K., Sedela L., Petite H. A biodegradable fibrin scaffold for mesenchymal stem cell transplantation. Biomaterials 2003; 24(14): 2497-2502, https://doi.org/10.1016/s01429612(02)00618-x.

12. Dikovsky D., Bianco-Peled H., Seliktar D. The effect of structural alterations of PEG-fibrinogen hydrogel scaffolds on 3-D cellular morphology and cellular migration.
Biomaterials 2006; 27(8): 1496-1506, https://doi.org/10.1016/j. biomaterials.2005.09.038.

13. Hughes C.S., Postovit L.M., Lajoie G. Matrigel: a complex protein mixture required for optimal growth of cell culture. Proteomics 2010; 10(9): 1886-1890, https://doi. org/10.1002/pmic.200900758.

14. Veronese F.M. Peptide and protein PEGylation: a review of problems and solutions. Biomaterials 2001; 22(5): 405-417, https://doi.org/10.1016/s0142-9612(00) 00193-9.

15. Zhang G., Wang X., Wang Z., Zhang J., Suggs L. A PEGylated fibrin patch for mesenchymal stem cell delivery. Tissue Eng 2006; 12(1): 9-19, https://doi.org/10.1089/ ten.2006.12.9. 